\title{
CRESCIMENTO DE ESPÉCIES NATIVAS DA AMAZÔNIA SUBMETIDAS AO PLANTIO NO ESTADO DE RORAIMA
}

\author{
GROWTH OF AMAZON NATIVE SPECIES SUBMITTED TO THE PLANTATION IN THE RORAIMA \\ STATE
}

Helio Tonini ${ }^{1}$ Moisés Mourão Cordeiro de Oliveira Junior ${ }^{2}$ Dalton Schwengber ${ }^{3}$

RESUMO

Um importante desafio para a pesquisa florestal na Amazônia é o de encontrar formas de reflorestar áreas degradadas com a utilização de um maior número de espécies nativas e identificar espécies tropicais comercialmente atrativas adaptadas a plantios a pleno sol. Este trabalho teve como objetivo avaliar o crescimento inicial em diâmetro e altura de seis espécies nativas da Amazônia em um ensaio preliminar de espécies. Os dados para a realização deste estudo foram oriundos de remedições de 72 árvores em um período de 9 anos. As espécies selecionadas para este trabalho foram a cupiúba (Goupia glabra), o cumaru (Dipterix odorata), a andiroba (Carapa guianensis), a castanheira-do-brasil (Bertholletia excelsa), o parápará (Jacaranda copaia) e a tatajuba (Bagassa guianensis). As curvas cumulativas de crescimento em diâmetro e altura foram obtidas pelo ajuste da função de Chapman-Richards. Apesar da pouca idade dos plantios, obteve-se bom ajuste da função para as espécies estudadas, sendo o pará-pará a espécie que apresentou maior crescimento em diâmetro e altura em todas as idades. A análise do incremento médio anual em diâmetro indicou que, com exceção da cupiúba, pode-se esperar incrementos médios superiores a $2 \mathrm{~cm}$, mediante a adoção de espaçamentos e programas de desbaste adequados.

Palavras-chave: reflorestamento; curva de crescimento; função de Chapman-Richards.

\begin{abstract}
An important forest research challenge in the Amazonian is finding forms of reforesting degraded areas with the use of a larger number of native species and identify tropical species commercially attractive adapted to clear-cut areas. This work had as objectives to evaluate the initial growth in diameter and height of six native Amazonian species in a preliminary species trial. The data were obtained from measures of 72 trees 9 years after planting. The selected species for this study were cupiúba (Goupia glabra), cumaru (Dipterix odorata), andiroba (Carapa guianensis), brazil nut (Bertholletia excelsa), pará-pará (Jacaranda copaia) and tatajuba (Bagassa guianensis). The cumulative growth curves for diameter and height was obtained by the Chapman - Richards function. In spite of the low age of the stands, it was obtained good fit to the function for the studied species. Pará-pará (Jacaranda copaia), presented best diameter and height growth in all the ages. The diameter mean annual increment analysis showed that, except for the cupiúba (Goupia glabra), can be expected increments larger than $2 \mathrm{~cm}$, by appropiate spacings and thinning.
\end{abstract}

Keywords: reforestation; growth curve; Chapman-Richards function.

\section{INTRODUÇÃO}

Segundo dados publicados pela FAO (2005), a área total de florestas no mundo é de 4 bilhões de hectares. O desflorestamento mundial, sobretudo, a conversão de florestas em terras agricultáveis é de 13 milhões de hectares por ano, e, atualmente, a América do Sul é o continente com as maiores perdas em área florestal, cerca de 4,3 milhões de hectares anuais.

As florestas tropicais fornecem várias espécies de alto valor comercial para o mercado internacional. Teoricamente, essas florestas deveriam fornecer, via manejo florestal, uma produção sustentada dessa matéria-prima. Porém, na prática, isto não ocorre em conseqüência de uma série de razões consideradas de difícil solução a curto prazo (ERSKINE et al., 2005).

Até o ano 2000, o desflorestamento na Amazônia foi de 59 milhões de hectares, o que corresponde à

1. Engenheiro Florestal, Dr., Pesquisador da Embrapa Roraima, BR 174, km 8, Caixa Postal 133, CEP 69301-970, Boa Vista (RR). helio@cpafrr.embrapa.br

2. Engenheiro Florestal, Dr., Pesquisador da Embrapa Roraima, BR 174, km 8, Caixa Postal 133, CEP 69301-970, Boa Vista (RR). mmourão@cpafrr.embrapa.br

3. Engenheiro Florestal, Dr., Pesquisador da Embrapa Roraima, BR 174, km 8, Caixa Postal 133, CEP 69301-970, Boa Vista (RR).dalton@cpafrr.embrapa.br

Recebido para publicação em 19/10/2006 e aceito em 31/05/2007. 
cerca de $15 \%$ da área total. Dessa área, cerca de 18 milhões de hectares foram transformados em pastagens, existindo estimativas de que pelo menos a metade dessa área estaria atualmente degradada ou em estado de degradação (GEO BRAZIL, 2002)

O reflorestamento de parte dessas áreas, além dos benefícios ecológicos, aumentaria a oferta de madeira reflorestada na região, aumentando a renda na propriedade rural e diminuindo a pressão sobre as florestas naturais remanescentes. A inclusão do elemento florestal entre as atividades nas propriedades rurais oferece não só a oportunidade de explorar novas fontes de renda, mas também de tornar produtivas áreas consideradas marginais para as culturas agrícolas tradicionais (SHIMIZU, 1998).

No entanto, o reflorestamento de áreas degradadas com a utilização apenas de espécies exóticas altamente produtivas, como os Pinus, os Eucalyptus e as Acacias, não promove a reposição da madeira de alto valor comercial oriunda de espécies nativas e pode resultar em uma simplificação biológica e de uso do solo na região (ERSKINE et al., 2005).

Para Butterfield (1980), a exploração da flora nativa em reflorestamentos deveria ser implementada, pois aumentaria a diversidade de espécies utilizadas, reduzindo riscos biológicos e econômicos, pelo cultivo de uma grande variedade de espécies em sítios específicos, sob diferentes formas de plantio: puros, mistos, linhas quebra-vento e sistemas agroflorestais.

No entanto, a carência de conhecimentos científicos sobre o comportamento e o crescimento das espécies nativas nas diferentes condições edafo-climáticas da região amazônica e a baixa disponibilidade de sementes de boa qualidade são apontadas como dificuldades para o aumento da área reflorestada.

Um importante desafio para a pesquisa florestal na Amazônia é o de encontrar formas de reflorestar áreas degradadas com a utilização de um maior número de espécies nativas e identificar entre as várias espécies tropicais comercialmente atrativas quais são as melhores adaptadas a plantios a pleno sol.

Kanashiro e Yared (1988) relatam a implantação de vários ensaios comparativos de espécies a pleno sol e em condições de sombra parcial, visando a seleção de espécies nativas e exóticas para a região do Tapajós (PA). Estudos mais recentes, como os relizados por Deus et al. (1993), Franke et al. (2000), Piña Rodriques et al. (2000), Miranda e Valentim (2000), Souza et al. (2003) e Tonini et al. (2006), relataram o desempenho de espécies florestais para plantios na Amazônia.

Segundo Burley e Woods (1979), os ensaios de espécies e procedências devem ser programados em duas fases, a fase eliminatória e a de comprovação de espécies. Na fase eliminatória, testa-se uma grande quantidade de espécies, dependendo dos recursos e tempo disponível, em parcelas pequenas, por um curto período de tempo, visando a determinar a sobrevivência e o crescimento inicial. Na fase de comprovação, o objetivo é verificar, em condições normais de plantio, a superioridade de umas poucas espécies selecionadas nas fases anteriores.

Uma vez que informações sobre o crescimento de espécies nativas são escassas na Amazônia e praticamente inexistentes em Roraima, este trabalho foi realizado com o objetivo de analisar o crescimento em altura e diâmetro de espécies nativas em um ensaio preliminar realizado em área de floresta de transição no estado de Roraima.

\section{MATERIAL E MÉTODO}

A partir de 1995, foi iniciado, pela Embrapa Roraima, o plantio de uma coleção de espécies arbóreas nativas e exóticas em forma de arboretos, com a finalidade de avaliar preliminarmente espécies potenciais para sistemas agroflorestais e programas de reposição florestal. A área experimental localiza-se no Campo Experimental Confiança, no município do Cantá, distante cerca de $90 \mathrm{~km}$ de Boa Vista.

O clima na região é classificado como do tipo Ami (Köppen), com precipitação média anual de 1900 $\mathrm{mm}$, em que a época chuvosa ocorre no período de abril a setembro, sendo o mês de junho o mais chuvoso, representando cerca de 19\% da precipitação total anual (Mourão Junior et al., 2003).

O solo é classificado como do tipo Argissolo Vermelho Amarelo Distrófico, constituído por material mineral que tem como características argila de atividade baixa e horizonte B textural.

Os dados para a realização deste estudo foram oriundos de remedições de 72 árvores em um período 
de 9 anos. As espécies selecionadas para este trabalho foram a cupiúba (Goupia glabra), o cumaru (Dipterix odorata), a andiroba (Carapa guianensis), a castanheira-do-brasil (Bertholletia excelsa), o pará-pará (Jacaranda copaia) e a tatajuba (Bagassa guianensis), todas consideradas de boa aceitação no mercado nacional e internacional, com boa forma do fuste e taxa de crescimento em plantios a pleno sol nas condições Amazônicas. As principais características das espécies estudadas são apresentadas na Tabela 1.

TABELA 1: Características das espécies avaliadas.

TABLE 1: Characteristics of the evaluated species.

\begin{tabular}{|c|c|c|c|c|c|}
\hline Espécie & Família & S (\%) & $\begin{array}{c}\text { Densidade da } \\
\operatorname{Madeira}^{2}\left(\mathrm{~g} / \mathrm{cm}^{3}\right)\end{array}$ & $\begin{array}{c}\text { Grupo } \\
\text { Ecológico }^{1}\end{array}$ & $\begin{array}{l}\text { Utilização da } \\
\text { Madeira }^{2}\end{array}$ \\
\hline $\begin{array}{l}\text { Pará-pará } \\
\text { (Jacaranda copaia) }\end{array}$ & Bignoniaceae & 80 & $0,38-0,40$ & $\mathrm{P}$ & $\begin{array}{l}\text { Caixas, brinquedos, } \\
\text { compensados, } \\
\text { marcenaria }\end{array}$ \\
\hline $\begin{array}{l}\text { Castanheira } \\
\text { (Bertholletia } \\
\text { excelsa) }\end{array}$ & Lecythidaceae & 80 & $0,70-0,75$ & DL & $\begin{array}{l}\text { Forros, vigas, } \\
\text { carpintaria }\end{array}$ \\
\hline $\begin{array}{l}\text { Cumaru } \\
\text { (Dipterix odorata) }\end{array}$ & $\begin{array}{l}\text { Leguminosae- } \\
\text { papilionoideae }\end{array}$ & 92 & $0,91-1,0$ & DL & $\begin{array}{l}\text { Implementos agrícolas, } \\
\text { dormentes, construção } \\
\text { naval, moirões }\end{array}$ \\
\hline $\begin{array}{l}\text { Tatajuba } \\
\text { (Bagassa } \\
\text { guianensis) }\end{array}$ & Moraceae & 100 & $0,75-0,85$ & $\mathrm{P}$ & $\begin{array}{l}\text { Carpintaria, dormentes, } \\
\text { contrução civil e naval, } \\
\text { marcenaria }\end{array}$ \\
\hline $\begin{array}{l}\text { Andiroba } \\
\text { (Carapa } \\
\text { guianensis) }\end{array}$ & Meliaceae & 76 & $0,70-0,75$ & TS & $\begin{array}{l}\text { Carpintaria, } \\
\text { marcenaria, construção } \\
\text { civil e naval, esquadrias }\end{array}$ \\
\hline $\begin{array}{l}\text { Cupiúba } \\
\text { (Goupia glabra) }\end{array}$ & Celastraceae & 48 & $0,80-0,90$ & $\mathrm{P}$ & $\begin{array}{l}\text { Dormentes, soalhos, } \\
\text { marcenaria, caixas, } \\
\text { caibros, carpintaria }\end{array}$ \\
\hline
\end{tabular}

Em que: 1 = Lopes et al. (2001) e Jennings et al. (2001); 2 = Loureiro et al. (1979 a e b); S (\%) = sobrevivência em porcentagem; $\mathrm{P}=$ pioneira; $\mathrm{DL}=$ demandante por luz; $\mathrm{TS}=$ tolerante à sombra.

Os plantios foram feitos em parcelas retangulares de $180 \mathrm{~m}^{2}$, compostas por cinco linhas com seis plantas, localizadas próximas, de forma a não existirem grandes variações de sítios. O espaçamento adotado foi o de 2,5 x 2 metros, totalizando trinta plantas por espécie. Os plantios foram feitos por coveamento direto, com a aplicação de $60 \mathrm{~g}$ de superfosfato triplo na cova. Anualmente, foram medidos a circunferência tomada a 1,30 m do solo (CAP) e a altura total (h) nas três linhas centrais (12 plantas úteis), com a utilização de fita métrica e hipsômetro Blume Leiss.

A curva cumulativa de crescimento foi obtida pelo ajuste da função de Chapman-Richards. Essa função tem sido amplamente utilizada na modelagem de fenômenos biológicos devido a sua acuracidade e flexibilidade, sendo a mais utilizada em estudos de crescimento na área florestal (ZEIDE, 1993). Usualmente, é expressa na forma:

$$
y=A\left(1-E X P^{(-K \times t)}\right)^{R}
$$

Em que: $\mathrm{y}=$ variável dependente ; $\mathrm{A}=$ assíntota; $\mathrm{K}=$ parâmetro relacionado à taxa de crescimento; $\mathrm{R}=$ parâmetro de forma; $\mathrm{t}=$ idade (anos).

Para o ajuste dessa função, utilizou-se o algoritmo proposto por Marquardt. Para assegurar que a verdadeira solução foi encontrada, após a convergência, repetiram-se os valores obtidos no primeiro processamento até a coincidência. A qualidade do ajuste foi avaliada pela análise do coeficiente de determinação, erro-padrão de estimativa e distribuição dos resíduos. A significância estatística dos parâmetros estimados foi determinada pela observação do intervalo de confiança assintótico para $95 \%$ de probabilidade de confiança. Dessa forma, a hipótese nula $\mathrm{H}_{0}: \mathrm{b}_{\mathrm{j}}=\mathrm{O}$ foi rejeitada quando o intervalo não incluiu zero. Os contrastes entre os intervalos de confiança assintóticos foram utilizados para verificar diferenças estatísticas entre os parâmetros estimados por espécie. A curva do incremento médio anual (IMA) foi obtida partindo da razão entre os valores estimados pela função e a idade. 


\section{RESULTADOS E DISCUSSÃO}

O ajuste da função de Chapman-Richards para as espécies estudadas pode ser observado na Tabela 2. Observa-se a boa qualidade de ajuste da função, com altos valores para o coeficiente de determinação e baixos valores de erro padrão de estimativa. A análise gráfica da distribuição dos resíduos indicou que a função pode ser empregada sem tendenciosidade. De forma geral, os parâmetros mostraram-se todos significativos, excetuando-se o coeficiente $\mathrm{K}$ no ajuste da função para o crescimento em altura da castanheira-do-brasil e cupiúba.

Segundo Fekedulegn et al. (1999), a não-significância dos parâmetros estimados pode indicar que: um ou mais parâmetros na função podem não ser úteis, e uma nova parametrização, com um menor número de coeficientes, poderia ser mais apropriada; os dados utilizados no ajuste da função não permitem a utilização de todos os parâmetros; ou a concepção teórica do modelo não se ajusta ao sistema biológico modelado. Assim, consideramos que a ausência de significância deve-se à pouca idade dos plantios, de forma que a curva de crescimento para algumas espécie, como é o caso da castanheira-do-brasil, ainda não apresenta um padrão sigmoidal durante o período de mensuração.

Apesar dessa restrição, conforme Lei e Zhang (2004), a utilização de um modelo não-linear teórico apresenta a vantagem de possuir uma hipótese biológica associada a uma causa ou função do fenômeno descrito pela variável resposta. $\mathrm{O}$ emprego de uma função empírica, como um polinômio, não permite qualquer interpretação, por não possuir parâmetros de real significado sob a perspectiva florestal.

Por meio do contraste entre os intervalos de confiança assintóticos, observou-se que as curvas para o DAP e a altura diferiram apenas em sua assíntota (Tabela 2), indicando que o diâmetro final esperado é maior para o pará-pará e a castanheira-do-brasil, e a altura, para o pará-pará, a tatajuba e a castanheira-dobrasil. Segundo o critério de comparação utilizado, o ponto de inflexão e a taxa em que as espécies alcançam o valor esperado final não diferem entre si, indicando semelhança de crescimento.

TABELA 2: Coeficientes e erro padrão assintótico obtidos no ajuste da função de Chapman-Richards. TABLE 2: Coeficients and assintotic standart error to the Chapman-Richards function fit.

\begin{tabular}{|c|c|c|c|c|c|}
\hline \multicolumn{6}{|c|}{ Diâmetro } \\
\hline Espécie & A & $\mathrm{K}$ & $\mathrm{R}$ & $\mathrm{R}^{2}$ & Syx \\
\hline Andiroba & $11,05 \pm 0,27^{\mathrm{b}}$ & $0,73 \pm 0,16^{\mathrm{a}}$ & $2,96 \pm 1,17^{\mathrm{a}}$ & 0,74 & 1,28 \\
\hline Castanheira & $18,60 \pm 2,04^{\mathrm{ac}}$ & $0,63 \pm 0,12^{\mathrm{a}}$ & $0,91 \pm 0,36^{\mathrm{a}}$ & 0,64 & 3,37 \\
\hline Cumaru & $14,18 \pm 1,57^{b c}$ & $0,35 \pm 0,12^{\mathrm{a}}$ & $2,31 \pm 0,68^{\mathrm{a}}$ & 0,72 & 1,77 \\
\hline Cupiúba & $12,46 \pm 1,29^{b c}$ & $0,32 \pm 0,09^{\mathrm{a}}$ & $1,04 \pm 0,24^{\mathrm{a}}$ & 0,83 & 1,35 \\
\hline Pará-pará & $22,27 \pm 1,90^{\mathrm{a}}$ & $0,30 \pm 0,08^{\mathrm{a}}$ & $2,50 \pm 0,99^{\mathrm{a}}$ & 0,55 & 2,88 \\
\hline Tatajuba & $12,82 \pm 0,27^{\mathrm{b}}$ & $0,24 \pm 0,09^{\mathrm{a}}$ & $3,13 \pm 1,11^{\mathrm{a}}$ & 0,61 & 1,72 \\
\hline \multicolumn{6}{|c|}{ Altura } \\
\hline Espécie & $\mathrm{A}$ & $\mathrm{K}$ & $\mathrm{R}$ & $\mathrm{R}^{2}$ & Syx \\
\hline Andiroba & $12,10 \pm 1,0^{\mathrm{b}}$ & $0,38 \pm 0,08^{\mathrm{a}}$ & $1,59 \pm 0,41^{\mathrm{a}}$ & 0,79 & 1,20 \\
\hline Castanheira & $18,57 \pm 5,25^{\mathrm{a}}$ & $0,33 \pm 0,06^{\mathrm{a}}$ & $1,62 \pm 0,66^{\mathrm{a}}$ & 0,63 & 2,73 \\
\hline Cumaru & $13,57 \pm 0,56^{\mathrm{bc}}$ & $0,30 \pm 0,08^{\mathrm{a}}$ & $1,22 \pm 0,17^{\mathrm{a}}$ & 0,87 & 1,13 \\
\hline Cupiúba & $13,44 \pm 1,28^{\mathrm{bc}}$ & $0,28 \pm 0,08^{\mathrm{a}}$ & $1,32 \pm 0,48^{\mathrm{a}}$ & 0,73 & 1,31 \\
\hline Pará-pará & $19,59 \pm 1,27^{\mathrm{a}}$ & $0,20 \pm 0,12^{\mathrm{a}}$ & $1,46 \pm 0,36^{\mathrm{a}}$ & 0,78 & 1,90 \\
\hline Tatajuba & $16,16 \pm 0,89^{\mathrm{ac}}$ & $0,19 \pm 0,12^{\mathrm{a}}$ & $1,77 \pm 0,53^{\mathrm{a}}$ & 0,65 & 2,24 \\
\hline
\end{tabular}

Em que: $A, K, R$ = parâmetros da função de Chapman-Richards; $R^{2}=$ coeficiente de determinação; Syx = erro-padrão de estimativa.

Valores precedidos de mesma letra, na vertical, não diferem quanto ao contraste dos intervalos de confiança assintóticos dos coeficientes.

No entanto, observa-se, na Figura 1, que existem diferenças entre as espécies no padrão de crescimento em diâmetro e altura. As razões para essas diferenças foram discutidas por Husch et al. (1982), os quais afirmaram que, quando se trabalha com curvas de crescimento, deve-se considerar que cada espécie, 
ou mesmo cada árvore, necessita de um tempo próprio para a sua construção. Essetempo fisiológico varia com a espécie e o estágio de desenvolvimento.

Deve-se considerar que as florestas tropicais caracterizam-se pela ocorrência de um grande número de espécies com hábitos de crescimento distintos (VANCLAY, 1991) e que também existe uma série de fatores que podem influenciar nas diferenças de crescimento entre espécies. Entre esses fatores, a demanda por luz e a estratégia de regeneração estão diretamente relacionadas com a taxa potencial de crescimento (WHITMORE, 1990; SILVA et al., 1995; FINEGAN e CAMACHO, 1999).

Em uma floresta natural, existem espécies mais adaptadas a grandes (espécies pioneiras) e pequenas clareiras (espécies clímax). Além disso, as condições ambientais em uma plantação diferem das encontradas em uma floresta natural, estando mais próximas às encontradas em grandes clareiras (ERKINE et al., 2005). Portanto, é esperado que espécies de estágios sucessionais iniciais apresentem melhor desempenho de crescimento inicial, uma vez que as espécies nativas têm uma maior probabilidade de êxito quanto mais próximas estiverem as condições de plantio das condições naturais (KAGEYAMA e CASTRO, 1989).

A curva cumulativa de crescimento em diâmetro para todas as espécies durante 9 anos é apresentada na Figura 1 (A). Observa-se que o pará-pará apresentou superioridade de crescimento em todas as idades, atingindo um diâmetro médio de $20,7 \mathrm{~cm}$, aos 9 anos. A castanheira-do-brasil, apesar de apresentar crescimento inicial mais lento, atingiu maiores diâmetros a partir do quarto ano de idade em comparação com as demais espécies, as quais apresentaram um diâmetro médio de $16,7 \mathrm{~cm}$, aos 9 anos. A cupiúba foi a espécie de crescimento mais lento durante grande parte do período de observação, porém, a partir do oitavo ano, superou em desempenho a andiroba, atingindo $11,7 \mathrm{~cm}$, superando os $10,9 \mathrm{~cm}$, aos 9 anos.

Para o crescimento em altura (Figura $1 \mathrm{~B}$ ), observa-se superioridade para o pará-pará e a tatajuba em todas as idades, os quais atingiram alturas de $17,7 \mathrm{~m}$ e $15,2 \mathrm{~m}$, aos nove anos, respectivamente. A castanheira-do-brasil apresentou crescimento inicial mais lento, porém superou as demais espécies a partir do oitavo ano (13,7 m aos 9 anos). O pior desempenho foi observado para a cupiúba e a andiroba, com 9,7 e $10,7 \mathrm{~m}$ respectivamente.

A superioridade de crescimento apresentada pelo pará-pará reflete a sua estratégia de crescimento, apta à colonização de espaços abertos, e concorda com os resultados obtidos por Erskine et al. (2005), ao observarem que espécies de estágios sucessionais iniciais, com madeira de baixa densidade, apresentaram maior crescimento inicial.
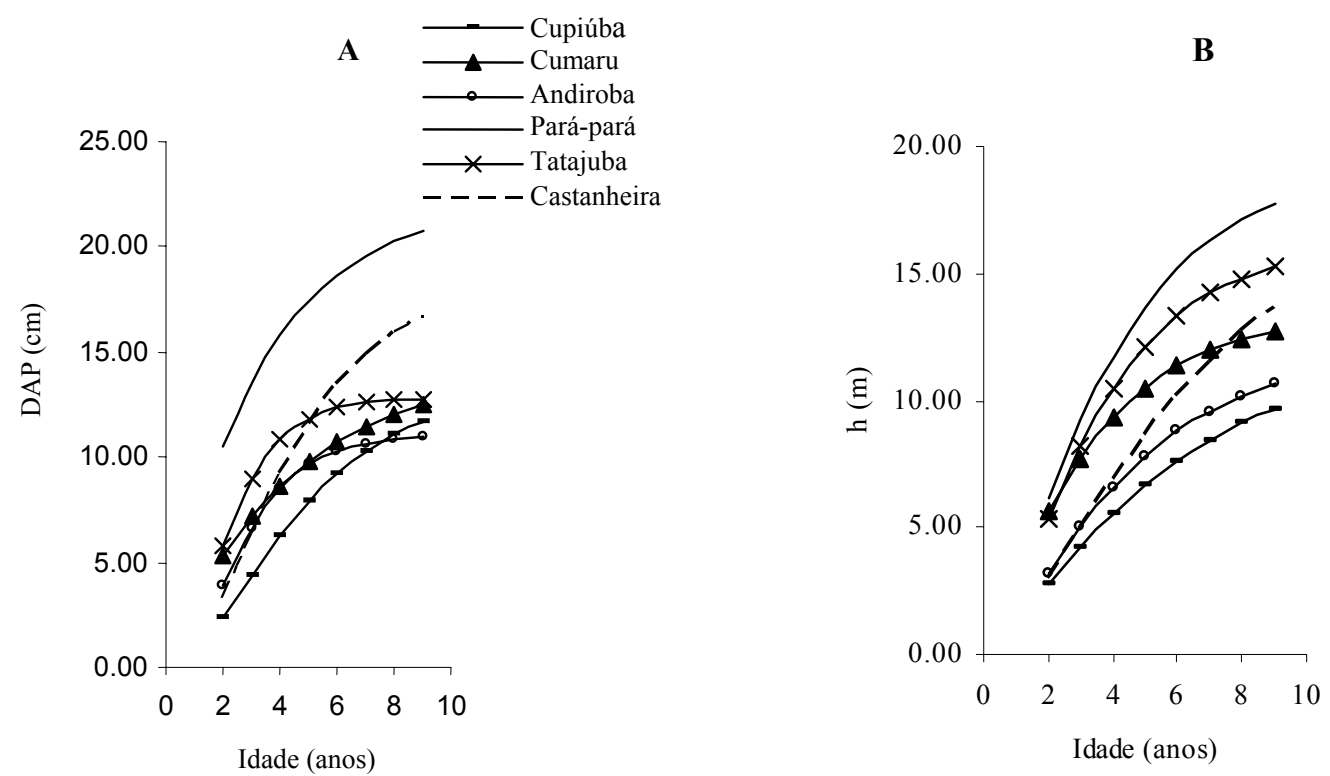

FIGURA 1: Curva de crescimento cumulativo para o DAP (A) e altura total (B).

FIGURE 1: Cumulative growth curves to DBH (A) and total height (B). 
A curva de incremento médio anual (Figura 2) mostra o crescimento explosivo do pará-pará nas idades iniciais, que é acompanhado por uma redução drástica já a partir do segundo ano, concordando com Whitmore (1990) e Kageiama e Castro (1989), ao afirmarem que as taxas de crescimento em altura são muito altas para espécies pioneiras em florestas tropicais, podendo, no entanto, apresentar um declínio vertiginoso no crescimento em idades mais avançadas. Espécies de estágios sucessionais mais avançados, com crescimento inicial mais lento, podem aumentar o ritmo com a idade. Essa tendência pode ser observada na melhora gradual de desempenho da castanheira-do-brasil, indicando que o crescimento inicial pode não caracterizar o potencial da espécie para plantações, exigindo ensaios de longa duração.

O tamanho alcançado por uma árvore em uma determinada idade relaciona-se com o espaço disponível para o seu crescimento, que aumenta com o tamanho e a idade (WADSWORTH, 2000). Na Figura 2, observa-se que todas as espécies apresentam tendência de redução no incremento médio em diâmetro e altura, podendo-se deduzir que está relacionada à competição por luz e nutrientes por causa do fechamento do dossel e à expansão do sistema radicular.
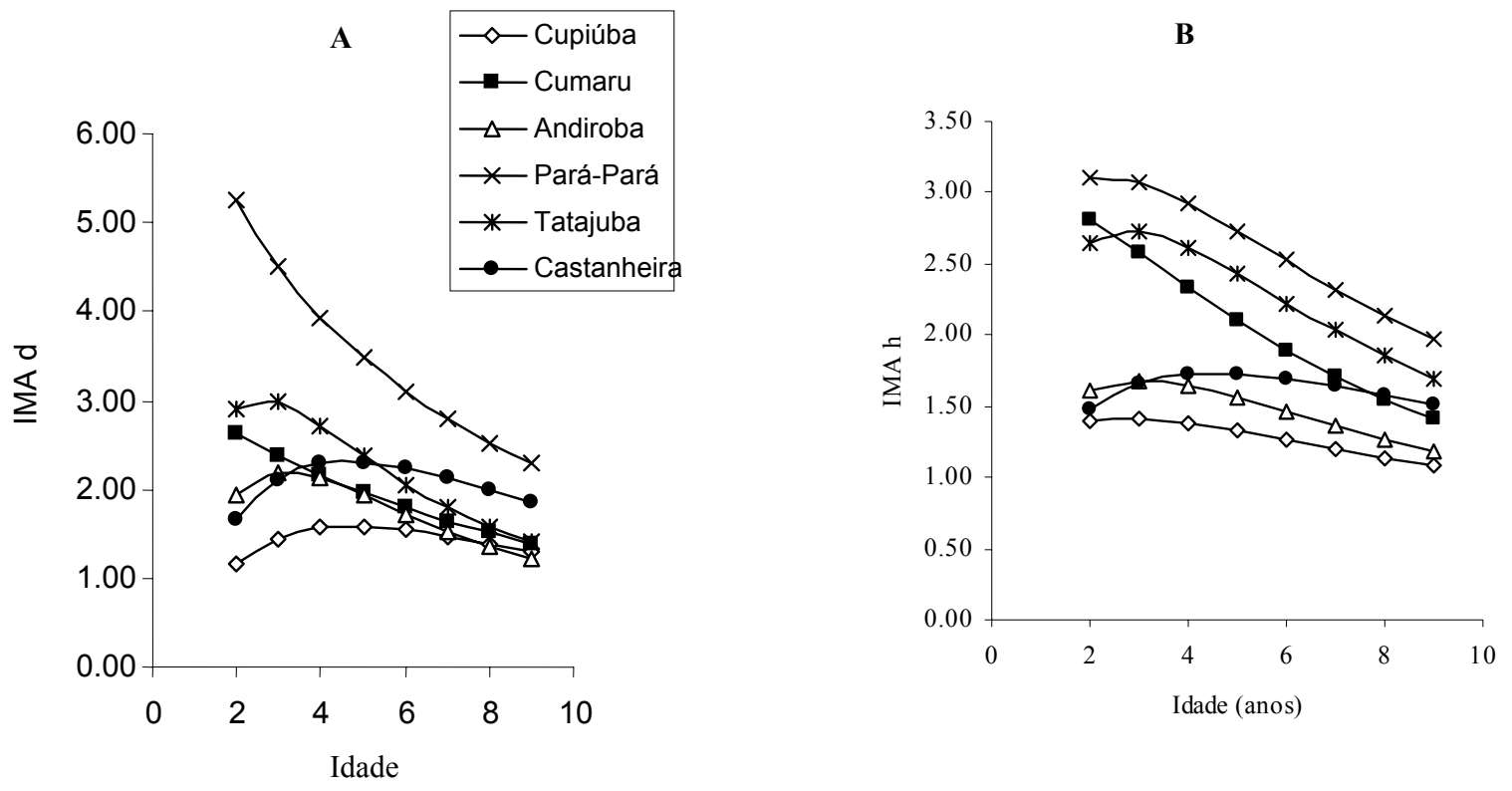

FIGURA 2: Incremento médio anual para o DAP (A) e altura (B) para as espécies estudadas.

FIGURE 2: Mean anual increment for DBH (A) and height (B) for study species.

A idade de culmínio do incremento médio anual variou com a espécie entre 2 a 5 anos, tanto para o diâmetro como para a altura. Quanto ao diâmetro, a idade foi de 2 anos para o cumaru $(2,6 \mathrm{~cm})$ e o pará-pará $(5 \mathrm{~cm}) ; 3$ anos para a tatajuba $(3,0 \mathrm{~cm}) ; 4$ anos para a andiroba $(2,1 \mathrm{~cm})$; e 5 anos para a castanheira $(2,3 \mathrm{~cm})$ e a cupiúba $(1,6 \mathrm{~cm})$.

Quanto à altura, a idade de culmínio foi de 2 anos para o cumaru $(2,8 \mathrm{~m})$ e o pará-pará $(3,1 \mathrm{~m}) ; 3$ anos para a cupiúba $(1,4 \mathrm{~m})$, andiroba $(1,7 \mathrm{~m})$ e tatajuba $(2,7 \mathrm{~m})$; e 5 anos para a castanheira $(1,7 \mathrm{~m})$.

O IMA representa a melhor estimativa da taxa máxima de crescimento, que pode ser mantida de forma contínua por uma determinada espécie em um determinado sítio (SMITH et al., 1997). Isso indica que, com exceção da cupiúba, pode-se esperar a manutenção de incrementos diamétricos superiores a $2 \mathrm{~cm}$ mediante a adoção de espaçamentos e programas de desbastes adequados. De forma geral, as espécies apresentaram crescimento promissor e altas taxas de sobrevivência, destacando-se o pará-pará, com o melhor desempenho, e a cupiúba, com o pior. 


\section{CONCLUSÕES}

Com os resultados obtidos neste estudo, pode-se concluir que:

a) A função de Chapman-Richards permitiu boas estimativas de crescimento em altura e diâmetro para as espécies estudadas.

b) Entre as espécies estudadas, o pará-pará (Jacaranda copaia) foi a espécie que apresentou maior crescimento em altura e diâmetro em todas as idades.

c) O Incremento médio anual em diâmetro indicou que, com exceção da cupiúba (Goupia glabra), pode-se esperar valores superiores a $2 \mathrm{~cm}$ por ano para as espécies estudadas mediante a adoção de espaçamentos e programas de desbaste adequados.

d) Pode-se recomendar todas as espécies avaliadas para a realização de ensaios rigorosos de preparo do solo, adubação e espaçamento em diferentes condições edafoclimáticas no estado de Roraima.

\section{REFERÊNCIAS BIBLIOGRÁFICAS}

BUTTERFIELD, R.P. Promoting biodiversity: advances in evaluating native species for reforestation. Forest Ecology and Management, v.75, p.111-121, 1995.

BURLEY, J.; WOOD, P.J. Manual sobre investigaciones de especies y procedencias con referencia especial a los tropicos. Londres: Commonwealth Forestry Institute/University of Oxford, 1979. 233 p.

DEUS, C. E.; WEIGAND JUNIOR, R.; KAGEIAMA, P.Y. et al. Comportamento de 28 espécies arbóreas tropicais sob diferentes regimes de luz em Rio Branco, Acre. Rio Branco: UFAC, 1993, 170 p.

ERSKINE, P.D.; LAMB, D.; BORSCHMANN, G. Growth performance and management of a mixed rainforest tree plantation. New Forest, v.29, p.117-234, 2005.

FAO. Global Forest resources. Assessment 2005. Progress towards sustainable forest management. Roma: FAO, 2006. $317 \mathrm{p}$.

FEKEDULEGN, D.; SIURTAIN.; M.P.M.; COLBERT, J.J. Parameter estimation of nonlinear growth models in forestry. Silva Fennica, v.33, n.4, p.327-336, 1999.

FRANKE, I.L.; MIRANDA, E.M.; VALENTIM, J.F. Comportamento de espécies arbóreas de uso múltiplo para sistemas agroflorestais no Estado do Acre. In: CONGRESSO BRASILEIRO DE SISTEMAS AGROFLORESTAIS, 3. 2000, Manaus. Anais.... Manaus: Embrapa Amazônia Ocidental, 2000, p.97-100.

FINEGAM, B., CAMACHO, M. Stand dynamics ina a logged and silviculturally treated Costa Rica rain Forest, 19881996. Forest Ecology and Management, v.121, p.177-189, 1999.

GEO Brasil 2002. Environmental outlooks in Brazil. Brasilia: IBAMA, 2002,445 p.

HUSCH, B.; MILLER, C.I.; BEERS, T.W. Forest mensuration. New York: J. Wiley, 1982, 401 p.

JENNINGS, S.B.; LOPES, J.C.A.; BROWN, N.D.; WHITMORE, T.C. Desempenho comparativo de mudas de espécies florestais em gradiente microclimático criado experimentalmente. In: SILVA, J.N.M.; CARVALHO, J.O.P.; YARED, J.A.G. A silvicultura na Amazônia Oriental. Belém: Embrapa Amazônia Oriental/ DFID, p.228-251, 2001.

KAGEIAMA, P.Y.; CASTRO, C.F.A. Sucessão secundária, estrutura genética e plantações de espécies arbóreas nativas. IPEF, v.41/42, p.83-93, 1989.

KANASHIRO, M; YARED, J.A.G. Espécies nativas e exóticas: comportamento silvicultural no Planalto dos Tapajós Pará. Documentos, Belém, n.49, 1988, 29 p.

LEI, Y.C.; ZHANG, S.Y.; Features and partial derivatives of Bertalanffy-Richards growth models in forestry. Non linear analysis: modelling and control, v.9, n.1, p.65-73, 2004.

LOPES, J.C.A.; WHITMORE, T.C.; BROWN, N.D.; JENNINGS, S.B. Efeito da exploração nas populações de mudas em uma floresta tropical úmida no município de Moju, PA. In: SILVA, J.N.M.; CARVALHO, J.O.P.; YARED, J.A.G. A silvicultura na Amazônia Oriental. Belém:Embrapa Amazônia Oriental/ DFID, p.203-225, 2001.

LOUREIRO, A.A.; SILVA, M.F.; ALENCAR, J.C. Essências madeireiras da Amazônia. v.1. Manaus: INPA, 1979, $243 \mathrm{p}$.

LOUREIRO, A.A.; SILVA, M.F.;ALENCAR, J.C. Essências madeireiras da Amazônia. v.2. Manaus: INPA, 1979, $187 \mathrm{p}$. 
MIRANDA, E M; VALENTIM, J.F. Desempenho de doze espécies arbóreas nativas e introduzidas com potencial de uso múltiplo no estado do Acre, Brasil. Acta Amazonica, v.30, n.3, p.471-480, 2000.

MOURÃO JR, M. ; XAUD, M. R. ; XAUD, H. A. M. ; MOURA NETO, M. A. ; ARCO VERDE, M. F. ; PEREIRA, P. R. V. S. ; TONINI, H. . Precipitação pluviométrica em áreas de transição savana-mata de Roraima: campos experimentais Serra da Prata e Confiança. Boa Vista: Embrapa Roraima, 2003 (Comunicado Técnico).

PIÑA-RODRIQUES, F.C.M; LELES, P.S.S; FERRAZ, C; SANTOS, E.M. Comportamento de paricá (Schizolobium amazonicum) e virola (Virola surinamesis) em plantios puros e mistos na Amazônia. In: SIMPOSIO INTERNACIONAL SOBRE ECOSSISTEMAS FLORESTAIS, 6, 2000, Porto Seguro. Anais...Rio de Janeiro: Biosfera. 2000. p.73-74.

SHIMIZU, J. Espécies não tradicionais para plantios com finalidades produtivas e ambientais: Silvicultura e usos. In: ESPÉCIES NÃO TRADICIONAIS PARA PLANTIOS COM FINALIDADES PRODUTIVAS E AMBIENTAIS, 1, 1998. Anais... Curitiba: Embrapa Florestas, 1998, p.64-71.

SMITH, D.M.; LARSON, B, C.; KELTY, M, J.; ASHTON, P.MARK. The practice of silviculture. Applied forest ecology. New York: J.Wiley, 1997, 517 p.

SILVA, J.N.M.; CARVALHO, J.O.P., LOPES, J.C..A.; et al. Growth and yeld of a tropical rain Forest in the Brazilian Amazon 13 years after logging. Forest Ecology and Management, v.71, n.3. p.267-274, 1995.

SOUZA, C.R.; ROSSI, L,M.B.; AZEVEDO; et al. Desempenho de espécies florestais potenciais para plantios na Amazônia central. In: CONGRESSO BRASILEIRO FLORESTAL, 6, 2003. Anais...., São Paulo:SBS/SBEF, 2003, p.1-12.

TONINI, H.; ARCO-VERDE, M.; SCHWENGBER, D.R.; et al. Avaliação de espécies florestais em área de mata no estado de Roraima. Cerne, v.12, n.1, p.8-18, 2006.

VANCLAY, J.K. Aggregating tree species to develop diameter increment equations for tropical rainforests. Forest Ecology and Management, v.47, p.143-168, 1991.

ZEIDE, B.; Analysis of growth equations. Forest Science, v.39, n.3, p.594-616, 1993. 